\title{
ANÁLISE DAS INFORMAÇÕES CIENTÍFICAS UTILIZADAS NA GESTÃO DA INFORMAÇÃO DAS INSTITUIÇÕES DE ENSINO SUPERIOR SEGUNDO A LITERATURA INTERNACIONAL
}

ANALYSIS OF SCIENTIFIC INFORMATION USED IN THE INFORMATION MANAGEMENT OF HIGHER EDUCATION INSTITUTIONS ACCORDING TO INTERNATIONAL LITERATURE

ANÁLISIS DE LA INFORMACIÓN CIENTÍFICA UTILIZADA EN LA GESTIÓN DE LA INFORMACIÓN DE LAS INSTITUCIONES DE ENSEÑANZA SUPERIOR SEGÚN LA LITERATURA INTERNACIONAL

1Tatiane Priscila Fortunato

${ }^{1}$ Adilson Luiz Pinto

Universidade Federal de Santa Catarina ${ }^{1}$

Correspondência

1Tatiane Priscila Fortunato

Universidade Federal de Santa Catarina

Florianópolis, SC - Brasil

Email: tatianefortunato@gmail.com

ORCID: http://orcid.org/0000-0003-2041-8044

Submetido em: $21 / 11 / 2017$

Aceito em: 29/12/2017

Publicado em: 22/01/2018

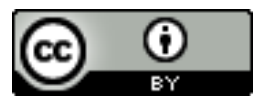

JITA: FJ. Knowledge management 
RESUMO: A abordagem se refere ao estudo da Gestão da Informação, no intuito de enfatizar sua relevância para a acessibilidade da informação e o desenvolvimento e disseminação do conhecimento nas diversas áreas de conhecimento. No que tange a Ciência da informação, dá-se a conexão com a organização do conhecimento, com a finalidade de demonstrar a importância dos indicadores científicos, coletados, processados e apresentados em literaturas internacionais disponíveis no Portal de Periódicos da Coordenação de Aperfeiçoamento de Pessoal de Nível Superior (CAPES). Com a finalidade de demonstrar a importância dos indicadores científicos no desempenho organizacional, faz-se o levantamento bibliográfico internacional, realizando uma análise minuciosa das informações dos estudos com apontamentos sobre os indicadores científicos $\mathrm{x}$ gestão da informação, utilizando a literatura em língua inglesa e espanhola, descrevendo a análise o Portfólio Bibliográfico selecionado, a ocorrência das pesquisas na área, a utilização de indicadores, a visão dos usuários e sua conexão com o ambiente. A proposta incide no levantamento dos dados e informações geridas neste contexto com vistas a colaborar com o mapeamento dos registros nas instituições de ensino superior, e estudiosos do tema, auxiliando na aceleração da gestão organizacional.

PALAVRAS-CHAVE: Gestão da Informação. Indicadores científicos. Índices científicos. Revisão da Literatura.

ABSTRACT: The approach refers to the study of Information Management, in order to emphasize its relevance for the accessibility of information and the development and dissemination of knowledge in the different areas of knowledge. With regard to Information Science, there is a connection with the organization of knowledge, with the purpose of demonstrating the importance of the scientific indicators, collected, processed and presented in international literature available in the Electronic Journal of the Coordenação de Aperfeiçoamento de Pessoal de Nível Superior (CAPES). In order to demonstrate the importance of scientific indicators in organizational performance, an international bibliographic survey is carried out, carrying out a detailed analysis of the information of the studies with notes on the scientific indicators versus information management, using the literature in English and Spanish, describing the analysis of the selected Portfolio Library, the occurrence of researches in the area, the use of indicators, the users' view and their connection with the environment. The proposal focuses on the collection of data and information managed in this context in order to collaborate with the mapping of records in higher education institutions and scholars, helping to accelerate of organizational management.

KEYWORDS: Information Management. Scientific indicators. Scientific indexes. Literature revision.

RESUMEN: El enfoque se refiere al estudio de la Gestión de la Información, con la finalidad de enfatizar su relevancia para la accesibilidad de la información, el desarrollo y la diseminación del conocimiento en las diversas áreas existentes. En lo que se refiere a la Ciencia de la información, se da la conexión con la organización del conocimiento, con la finalidad de demostrar la importancia de los indicadores científicos, recogidos, procesados y presentados en literaturas internacionales disponibles en el Portal de Revistas de la Coordenação de Aperfeiçoamento de Pessoal de Nível Superior (CAPES). Con el fin de demostrar la importancia de los indicadores científicos en el desempeño organizacional, se hace el levantamiento bibliográfico internacional, realizando un análisis minucioso de las informaciones de los estudios con apuntes sobre los indicadores científicos versus la gestión de la información, utilizando la literatura en lengua inglesa y española; describiendo el análisis del Portafolio Bibliográfico seleccionado; la ocurrencia de las investigaciones en el área; la utilización de indicadores; la visión de los usuarios, y; su conexión con el ambiente. La propuesta se centra en el levantamiento de los datos e informaciones gestionadas en este contexto con observación a colaborar con el mapeo de los registros en las instituciones de enseñanza superior, y estudiosos del tema, auxiliando en la aceleración de la gestión organizacional.

Palabras Clave: Gestión de la información. Indicadores científicos. Índices científicos. Revisión de literatura. 


\section{INTRODUÇÃ̃O}

A presente pesquisa tem como objetivo apresentar uma análise sobre a aplicação e/ou abordagens sobre a Gestão da Informação aliada aos indicadores científicos, produzida no campo da Ciência da Informação, tendo como finalidade mostrar a importância deste tipo de indicador como ferramenta de gestão e, como forma de organização do conhecimento das instituições de ensino, bem como situações específicas como as produções acadêmicas.

A investigação nos mostrou a relevância dos estudos voltados a área diante a ausência de trabalhos específicos referentes à indicadores científicos, e uma sistematização da recuperação de tais informações. Desta forma buscou realizar uma revisão sistematizada para nortear o trabalho.

A apresentação de indicadores no cenário científico se demonstra como uma importante fonte de informação no norteamento de tomadas de decisões (SPINAK,1998). Diante do exposto busca-se resolver a seguinte problemática: Existem aplicações práticas e estudos científicos que demonstrem os indicadores cientícos no auxílio da gestão da informação em instituições de ensino?

Neste sentido, como objetivo geral, foi realizada uma análise minuciosa das literaturas internacionais, nos idiomas inglês e espanhol para identificar a ocorrência de pesquisas que demonstrem o uso de indicadores científicos no auxílio da gestão da informação em instituições de ensino.

Para o estudo, toda a análise e investigação no que tange o tema Gestão da informação: indicadores científicos foram utilizados buscas e filtros em 05 (cinco) bases de dados disponíveis no Portal de Periódicos da CAPES, no campo da Ciência da Informação referente à seleção, identificação, análise e reflexão das literaturas publicadas nestes recursos.

O artigo está baseado em: introdução, referencial teórico, metodologia utilizada para o desenvolvimento do estudo, resultados e análises da pesquisa, considerações finais contribuições e sugestões para futuros estudos e, referências bibliográficas.

A gestão da informação pode ser aplicada e gerenciada por diversas áreas de conhecimento, por esta observação o estudo foi aplicado a Indicadores Científicos e Gestão da Informação devido ao fato de serem temas de interesse recente no campo, porém que são estudados desde a década 1970 no Brasil.

A gestão da informação é considerada como a ação sistêmica de procurar e entender as necessidades informacionais de uma organização e disponibilizá-las para a solução de problemas organizacionais. Quando gerenciados adequadamente, os dados se tornam em 
informações que servirão como base para as decisões empresariais (PORTAL EDUCAÇÃO, 2012).

A gestão da informação e organização do conhecimento tem sido estudada em diversas perspectivas, e estudos têm apontado novas áreas de abrangência como auxiliadores no desempenho organizacional, no qual visa à organização, acompanhamento, planejamento, revisão e cumprimento do papel social das organizações.

A organização do conhecimento é a ciência que ordena a estruturação e sistematização dos conceitos, de acordo com suas características, que podem ser definidas como elementos de herança do objeto, e a aplicação dos conceitos e classes dos conceitos ordenados pela indicação de valores, dos referentes conteúdos dos objetos ou assuntos (DAHLBERG, 2006, p.13).

Porém, o que vamos observar se refere à incipiência dos estudos na área de Gestão da informação relacionada ou aplicada ao contexto referente aos indicadores científicos no que tange o apoio administrativo, ou em caráter de medidas de desempenho das organizações de ensino.

Para o desenvolvimento sugere-se um olhar mais crítico ao ambiente, a distinção dos indicadores e medidas à atividade específica, visto o envolvimento de decisores, usuários, instituições e toda atividade nelas geridas.

Destaca-se também o alinhamento entre a decisão e ação, adaptando essas novas atividades à estratégia já existente (MICHELI; MANZONI, 2010), importante que essas medidas tenham conexão com a ação e, posteriormente, com a ação de gestão (OTLEY, 2001).

O desenvolvimento organizacional, seja ele por entidades privadas ou públicas, tem sido crescente no Brasil, já que o volume de informações para o desenvolvimento organizacional é extremamente relevante para a sobrevivência empresarial. Frente a este contexto, os investimentos das organizações nas atividades são (i) coleta, (ii) armazenamento, (iii) processamento dos dados e (iv) sua relação informacional. Estas ações são crescentes no processo e esta afirmação se traduz em muitos estudos abordados através da ciência e gestão da informação. O volume das informações organizacionais bem como sua qualidade percebida pelos gestores e colaboradores envolvidos passa a não ser mais somente com olhares puramente gerencial ou de produção, mas com características estratégicas que poupa tempo, custos, equipamentos, pessoas através do potencial informacional.

Tendo esta pesquisa, a intenção de investigar as práticas da gestão da informação relacionadas, e detectar os indicadores científicos contidos na literatura internacional, o que poderá auxiliar na resolução ou visualização de problemas da área e no processo de gestão organizacional. 


\section{GESTÃO DA INFORMAÇÃ̃}

Com o avanço tecnológico atrelado a Tecnologia da Informação, sistemas informacionais e de processamento de dados houve, a facilitação das questões organizacionais, humanas, estratégicas e comportamentais que envolvem as entidades públicas e privadas.

No entanto, a gestão e clareza da utilidade destas informações se tornam cada dia mais importante para que as informações de uso estratégico sejam eficazes e não se dispersem da missão da organização, num todo, ou mesmo quando aplicadas a departamentos ou atividades específicas.

De Sordi (2008) salienta que a lógica dos negócios atuais é que a gestão de dados disponíveis na organização, bem como daqueles passíveis de serem obtidos por parcerias e associações, pode tornar-se um importante recurso estratégico se planejados, coordenados e monitorados pela organização. Evidentemente, quanto maior o desafio almejado maior deverá ser o preparo da organização no que tange aos recursos: dados, informação e conhecimento.

O que tem se notado nas organizações, é que com seu desenvolvimento, as atividades são divididas por departamentos, quais podem ser de produção, marketing, recursos humanos, comercial, financeiros entre outros, obviamente de acordo com a atividade desempenhada pela organização. Porém, esta subdivisão, a ocorrência de informações sem conexões, ou seja, cada departamento gere seus objetivos e metas e, em sua maioria não se dá a observância que tais informações poderão ser relevantes não somente em seu departamento, qual também poderá ser a mesma na gestão de outros departamentos ou atividades.

A intenção da abordagem do estudo da gestão da informação é que haja a integração das informações entre os departamentos internos da organização e também com seus stakeholders para que não ocorra à duplicidade informacional e com isso o acúmulo de arquivos; a atualização das informações através desta colaboração dando mais valia a estes conteúdos, havendo a otimização do tempo visto sua acessibilidade com a informação compartilhada; a agilidade para tomada de decisões; a melhoria de processos em função da visualização de todo seu contexto; e consequentemente decisões estratégicas mais assertivas para o negócio.

Sobre a história e a evolução passamos por alguns períodos como a Revolução Industrial, o desenvolvimento da Era Tecnológica e, atualmente vivemos a chamada Era da Informação, no qual a cada dia somos nutridos de uma imensidão de informações que chegam até as pessoas de diversas formas, sejam orais, escritas, coletadas através de documentos, imagens, por mídias, sistemas, por sons e etc. 
O fato é que estamos num processo de transmissão e produção de informações constante, quais se referem ao nosso passado, presente e futuro e, este volume informacional deve ser direcionado, filtrado, concentrado e desenvolvido a ponto de gerarmos informações construtivas que tragam a evolução das áreas de conhecimento e das diversas formas de atuação e melhoria empresarial. Aqui, uma análise da gestão da informação como fonte de esclarecimento e desenvolvimento da organização do conhecimento das instituições, para que não ocorra simplesmente o acúmulo informacional e retrabalho organizacional e sim, a importância da informação no processo da gestão científica.

A Ciência da Informação está alinhada com diversas áreas do conhecimento e, nesta abordagem, apresenta-se aos indicadores científicos e sua conexão com as instituições de ensino, e a gestão das informações geridas para este fim.

Neste sentido, considerar a informação, sua proveniência e relevância têm sido preponderante para as organizações. Vivemos na sociedade da informação, entretanto, um progresso real somente irá ocorrer para aquelas organizações que perceberem e assimilarem a nova sociedade da informação (RODRIGUEZ; FERRANTE, 1995).

O compartilhamento das informações, sua gerência, organização e estrutura apresentam-se como papeis da Gestão da Informação, no qual se faz necessária para o atingimento dos objetivos organizacionais. A contínua expansão dos dados que circulam pelas organizações, abrangendo dados cadastrais de clientes, parceiros, fornecedores, funcionários, materiais, de produtividade, ativos e, principalmente, das transações destes com a organização, possibilita aos gestores obter informações e conhecimentos úteis, seja para a melhoria dos atuais produtos e serviços, seja para projeto e lançamento de novos produtos e serviços, permitindo, inclusive, o ingresso da organização em novos segmentos de negócio, de forma distinta daqueles já estabelecidos no segmento (DE SORDI, 2008).

Somos movidos por nossas informações e, não diferentemente as organizações, saber utilizar e direcionar tais informações às fontes adequadas se torna um recurso de melhoria. A gestão da informação apoia todo o contexto organizacional, aos seus departamentos ou subsistemas, auxiliando os gestores para tomada de decisões, possibilitando verificar sua situação no mercado onde está inserida, a comunicação entre os envolvidos interna e externamente e a sobrevivência.

Para Gameiro (1994) a informação é a fonte ordenadora da decisão, que é o alicerce da administração, consequentemente responsável pelo sucesso dos empreendimentos. A gestão da informação pode ou não estar atrelada a tecnologia, esta distinção aparece na medida em que se faz necessária à aplicação do recurso tecnológico para a agilidade da gestão.

Sua finalidade é apoiar e facilitar a comunicação entre os níveis tático, estratégico, operacional e seus stakeholders de acordo com a necessidade da organização proporcionando 
informações úteis no tempo correto para a gestão estratégica. Beal (2008) salienta que a Gestão da Informação está voltada para a coleta, o tratamento e a disponibilização da informação que dá suporte aos processos organizacionais tendo em vista o alcance de seus objetivos permanentes.

Desta maneira, a Gestão da Informação se concentra na busca, na identificação, na classificação, no processamento, no armazenamento e na disseminação de informações para que cheguem as pessoas e facilite a gestão dos recursos e insumos e a tomada de decisões.

Nesta abordagem, os indicadores científicos analisados pela gestão das organizações possuem uma maior assertividade nas ações estratégicas.

\subsection{Da análise dos indicadores / índices científicos}

Levando em consideração a participação dos indicadores, podendo ser compreendido por índices, para que se possa avaliar o desempenho no ambiente organizacional, identifica-se a sua ligação à função do planejamento estratégico da organização, no sentido de controlar, registrar e analisar as políticas institucionais, ações dos profissionais ou das organizações, auxiliando na organização e suas atividades de direção, controle, treinamento, manutenção e destinação de recursos entre outras atividades envolvidas.

Aos indicadores em sua abordagem nos faz perceber que busca conhecer e medir o desempenho dos indivíduos e da organização, estabelecendo uma comparação entre o desempenho esperado e o apresentado por indivíduos e/ou organizações, o que possibilita o desenvolvimento de programas de melhorias que possam diminuir ou até mesmo suprir deficiências organizacionais.

O manual Rationalization des Choix Budgetairies (RCB) editado pelo Governo Francês, define um indicador como:

\footnotetext{
um dado relativo a uma variável significativa que caracteriza um fenômeno e que serve de indicação para que se possa atuar sobre tal fenômeno. Ainda segundo esse manual, para se tirar o máximo proveito, a formulação e análise de indicadores devem ser articuladas em níveis segundo a sua utilização: de entrada, de saída, de estado e de gestão. Além dessas categorias, os indicadores ainda podem ser classificados em simples e complexos (SANCHES,1997).
}

Nesse contexto, o estudo dos indicadores científicos tem papel fundamental decorrente de sua intervenção na política de ação e normativas gerenciadas através das instituições de ensino. A observação destes indicadores, sua aplicação nas medidas de avaliação dos desempenhos institucionais, serve como um meio de se obter informações gerenciais e da sua efetividade qual permeia as atividades e funções de rendimento educacional, podendo também, ser utilizada para observar as deficiências e proporcionar políticas de melhoria 
institucional já que são capazes de nos demonstrar uma visualização do panorama a qual a análise está sendo aplicada.

Os índices e/ou indicadores são passíveis de ocorrer em toda a organização, com ou sem a utilização de métodos, onde qualquer observância de valor ou avaliação pode ser vista como um indicador, o importante é a especificação e conhecimento de qual fato ou situação estará em análise para que, estes indicadores estejam alinhados e proporcionem resultados assertivos.

A fim de atender as necessidades, as exigências, visualização e a sobrevivência das instituições, têm se tornado vital mecanismos que apresentem os indicadores como uma maneira de medir desempenhos.

A avaliação de desempenho deve ser capaz de assegurar a implementação da estratégia, influenciar positivamente o desempenho e identificar quando é necessário intervir com ações corretivas nas lacunas de desempenho significativas (NEELY et al., 1995; MELNYK et al., 2014), qual ajuda a identificar as causas do desempenho satisfatório ou insatisfatório, a contribuição de cada colaborador, promover o autodesenvolvimento e autoconhecimento e planos de ações corretivos. Desta forma Sink e Tuttle (1993, p.20),

Analisam o desempenho empresarial inter-relacionando sete critérios: eficiência, eficácia, qualidade, produtividade, qualidade de vida no trabalho, inovação e lucratividade, o que pode ser desmembrados como indicadores para futuras análises gerenciais no contexto das instituições de ensino superior.

Para fortalecer esse aspecto quantitativo é essencial agregar os estudos métricos, a fim de tornar possível outros aspectos das relações representadas por tais indicadores, como gráficos, densidades, centralidades, intermediações, proximidades, ocorrências e estimativas, o que se constitui na soma da cooperação entre dois ou mais pontos, ou simplesmente uma frequência científica projetada (PINTO et al, 2007).

No entanto, não têm o resultado almejado, à medida que as instituições desconhecem seus indicadores, o que poderá restringir a atuação e desempenho das instituições. Sua utilidade reforça as variáveis estratégicas, apoiando a gestão da informação, a organização do conhecimento, facilitando o entendimento de todo o processo e as estratégias internas e externas das instituições.

\section{PROCEDIMENTOS METOdOLógicos}

Nesta etapa trataremos do enquadramento metodológico da pesquisa, do instrumento de intervenção selecionado e dos procedimentos para coleta e análise dos dados. 


\subsection{Enquadramento metodológico}

Este estudo caracteriza-se exploratório, pois aprofundou o conhecimento acerca do problema pesquisado, gerando a reflexão e conhecimento quanto às delimitações do estudo, neste caso, as publicações a serem analisadas no Portfólio Bibliográfico. Em relação ao objetivo proposto classifica-se como exploratório e descritivo, pois demonstra as características dos dados encontrados nas publicações do Portfólio Bibliográfico.

O estudo é realizado com dados secundários, no caso os artigos selecionados para a composição do Portfólio Bibliográfico. O Portfólio Bibliográfico se traduz como sendo um instrumento de intervenção, qual exige que o pesquisador inicie o processo de explicitação dos conhecimentos que representam e tangenciam o tema, para tanto se utiliza as áreas de conhecimento que são denominadas os eixos de pesquisa, representadas por palavras-chave, passando por filtros de redundância, alinhamento ao título, alinhamento ao resumo e alinhados ao tema, originando assim um fragmento da literatura composto para a análise desta pesquisa.

No que se refere à abordagem do problema, sua característica é qualitativa, tendo por objetivo a análise das características encontradas nas publicações, na tentativa de identificar possíveis contribuições, sugestões e possíveis lacunas para aprofundar as pesquisas na área do conhecimento científico em estudo, respondendo assim, o problema de pesquisa.

Como método à pesquisa bibliográfica, visto que permite aos investigadores selecionar e analisar as publicações do Portfólio Bibliográfico proveniente da base de dados do Portal de Periódicos da CAPES, identificando a produção acerca do tema em estudo.

\subsection{Procedimentos para Coleta e Análise dos Dados}

Para o alcance do objetivo, foi revisado o fragmento da literatura, que trata da Gestão da informação: indicadores científicos, sendo necessária a seleção do Portfólio Bibliográfico. Como instrumento de intervenção para a coleta de dados, neste estudo foi utilizado uma revisão sistemática da literatura nas bases internacionais, que auxilia os pesquisadores na seleção e análise da literatura científica, frente a seus interesses, escolhas, delimitações e objetivos.

Para a análise, foi percorrida a seleção do Portfólio Bibliográfico, a fim de auxiliar o pesquisador, aprofundar e fundamentar a pesquisa quanto à relevância científica.

Após a identificação e verificação das abordagens internacionais, Portfólio Bibliográfico, e as práticas descritas quanto à temática Gestão da Informação: indicadores científicos; será possível mensurar a relevância do tema, realizar uma análise crítica e 
identificar lacunas na literatura referente ao tema, a fim de propor desafios para futuros trabalhos e objetivos de pesquisa.

A coleta de dados teve sua ocorrência a partir da seleção de um Portfólio Bibliográfico. Tendo para a seleção a definição de 02 (dois) eixos de pesquisa caracterizando o comando de busca. Como características, os artigos foram somente no idioma inglês e espanhol, com a delimitação temporal entre os anos 2007 a 2010, utilizando 05 (cinco) bases de dados, sendo alinhados após a verificação do título, resumo e disponibilidade integral do artigo.

Do processo de coleta de dados, fica assim caracterizado:

\section{ENTRADAS}

Tema: Gestão da Informação: indicadores científicos

Eixo 01: Gestão da Informação

Eixo 02: Indicadores científicos / índices científicos / dimensões científicas

Comando de Busca: ("information management" AND "scientific indicators" OR "scientific indicator" OR "scientific indexes" OR "scientific dimension" OR "scientific dimensions" OR "scientific index").

Bases Consultadas: EBSCO, EMERALD, PROQUEST/LISA, SCOPUS e WEB OF SCIENCE.

Resultado: 600 publicações.

Não periódicos excluídos: 1 serial, 583 book session.

Número de publicações duplicadas: 1 duplicação.

Resultado bruto: 15 publicações.

Exclusão por falta de alinhamento do tema no título: 5 publicações.

Exclusão por falta de alinhamento do tema no resumo: 2 publicações.

Publicação não recuperada: 1 publicação.

Disponibilidade na íntegra e portfólio bibliográfico para o estudo: 07 publicações.

Logo, o Portfólio Bibliográfico sob o fragmento da literatura referente ao tema Gestão da Informação: indicadores científicos possui um portfólio contendo 07 (sete) artigos para a análise, possibilitando as análises finais na percepção dos seguintes critérios:

A. Formas utilizadas para visualização da produção científica na literatura internacional,

B. Contextos aplicados,

C. E, lacunas no ramo de pesquisa e as oportunidades para novas pesquisas.

\section{DAS ABORDAgenS NO PORTIFólio BIBLIOGRÁFICO}

Num estudo mais detalhado do Portfólio Bibliográfico, com a finalidade de identificar as características do conteúdo estudado foi realizada análise do conteúdo. 
Cada conteúdo estudado apresenta sua característica e finalidades que são peculiares e, neste descritivo faz-se a visualização das características analisadas e sua relação e participação para a aumento das avaliações e contribuições num contexto científico.

A primeira análise se refere ao artigo Acquisition of T-shaped expertise: an exploratory study disponível na base de dados EBSCO, sua abordagem explicita a expectativa de identificar os profissionais com características para o desempenho e solução de problemas complexos (política, cultura, tecnologia e dimensões científicas).

As argumentações levam em consideração o conhecimento interdisciplinar de especialistas, caracterizando estes especialistas em forma de ' $\mathrm{T}$ ', como sendo profissionais com ampla gama de conhecimentos, interação e aprendizagem. A análise ocorre através de um estudo exploratório em dois programas educacionais no período de janeiro a maio de 2015.

Qual aponta que programas estudados possuem conhecimento disciplinar em ciência, engenharia, tecnologia, e os campos da matemática, sendo demonstrado a consciência e compreensão de outros conhecimentos.

As análises dos dados foram realizadas através de pré e pós testes, com o mapeamento de conceitos destinados em 500 artigos, que possibilitou analisar a aprendizagem e aquisição do conhecimento dos alunos, sua a utilidade e limitações para avaliar conhecimentos e evolução ao longo do tempo. Segundo o artigo, o mapeamento conceito oferece resultados que são promissores, mas precisam ser analisados de forma mais robusta.

Também foi possível perceber, que os alunos reconhecem que a experiência múltipla (profissionais em forma de $\mathrm{T}$ ) expandem sua compreensão se as atividades aplicadas ocorrerem fora do laboratório e, expressou maior consciência quanto aos seus limites e experiência correlata a outras áreas de conhecimento.

Cita que a união de cientistas, engenheiros e outras áreas de conhecimento, poderão trabalhar com objetivos compartilhados e poderão apoiar e colaborar para resolver problemas mais complexos. Desta forma perícia em forma de $\mathrm{T}$, pode ser considerada como um conjunto de capacidades que permite integração do próprio conhecimento com o conhecimento dos outros. Onde:

Educadores e empregadores devem se engajar em um diálogo contínuo sobre a 'característica $\mathrm{T}$ ', a experiência e construção de ferramentas de avaliação adicionais que detectem diferenças entre esses inovadores, 'responsável' com experiência em forma de $\mathrm{T}$ e de pessoas com apenas o conhecimento tradicional disciplinar conjuntos à sua disposição (CONLEY, COLEMAN; DENHAM; FOLEY; GORMAN, 2017). 
As informações contidas nesta primeira análise, se demonstra como sendo complementar ao contexto científico, pois possibilita análises que traduzem uma maior capacidade de mensuração e associação das ciências. Porem, limita-se ao estudo de artigos e seus mapeamentos o que poderão limitar as análises e sua abrangência.

No que trata o segundo artigo, Scientific Comparison between Web of Science (WoS) and Google Scholar: A Study from the Most Representative Authors of Brazil disponível na base de dados PROQUEST/LISA, analisou e comparou à produtividade e os índices de citação dos 10 autores brasileiros mais aparentes na ISI, quais os autores o caracterizaram como os Top 10 Brasil.

A análise ocorreu através das citações dos autores na Web of Science e Google Scholar, através da utilização de índices científicos quantitativos e qualitativos. A visibilidade científica ocorreu com análise temporal de 05 anos, o que possibilitou identificar a citação, cocitação e rede de cooperação dos autores os quais foram demonstrados através de mapas, gráficos e tabelas.

Apresentou a importância das bases de dados para a produção científica e carência versus oferta nos países emergentes. E a semelhança na recuperação dos dados nas bases, quanto à: descrição bibliográfica, revisão por pares, acesso aberto, referências de outras publicações, como livros, cartas e científica resumos e índices de citação, das diferenças e similaridades. Suas demonstrações analíticas tiveram caráter qualitativo e quantitativo.

$\mathrm{O}$ artigo demonstrou a confiabilidade das bases de busca e seus fatores de desempenho sob uma perspectiva da produção científica dos pesquisadores mas, quando trata das análises científicas, estas não se restringem somente a citação e cocitação mais também poderão se estender a relações e redes de cooperação, localidades onde se percebe maiores índices de produção, formação e alinhamento destes profissionais entre outros. O que possibilidade um olhar mais crítico, as maiores possibilidades de estudos e aprofundamento dos critérios apontados não deixando se ser relevante pois é sim um forma a qual deve ser realizados levantamentos e estimativas pois, compõem o quadro de evolução institucional.

No que se refere a terceira análise, sobre o artigo Differentiating, Describing, and Visualizing Scientific Space: A Novel Approach to the Analysis of Published Scientific Abstracts disponível também na base de dados PROQUEST/LISA, os autores demonstram um entendimento que a ciência é um processo social e evolutivo, e trazem à tona a questão dos índice de citação como forma de investigar redes científicas e dinâmicas sociais.

Citam que os trabalhos submetidos são explorados e utilizados de acordo com vários procedimentos de análise e visualização que modelam o quadro científico através da utilização dos índices de citação, de suas temáticas, relações e cooperações ao invés de explorar o significado e semântica, a aplicação de análise semântica latente para a análise,

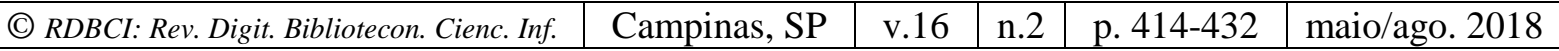


diferenciação e visualização de documentos científicos, quais, em seguida, apontam as bases como a forma e procedimento aplicados para estudar padrões de citação.

Chamam atenção a semântica latente, que segundo os autores, é capaz de caracterizar os temas dominantes dentro da ciência, agrupando artigos em conformidade com base em suas designações de campo o que possibilita visualizar os resultados de uma forma baseada em princípios, e permite representações visuais da relação entre artigos científicos e campos, apresentando benefícios para documentar indexação e sua potencial aplicação para análise das citações.

Esta, por sua vez, se baseia na teoria da semântica e da linguagem de aquisição desenvolvidos pelos psicolingüistas e cientistas da computação, o significado das palavras emergentes e as associações entre elas, sua coocorrência. $\mathrm{O}$ artigo também demonstra a utilidade do processo de diferenciação e visualização de um índice de artigos científicos sobre a base dos seus resumos, e, propõe maneiras em que a representação espacial resultante da ciência incorpore em análise de citação, representado em cada documento, em termos de um número de variáveis ou dimensões semântica através da construção de um termo-adocumento, um conjunto de dados multicampo.

Também demonstram quantitativamente que a diferenciação semântica latente é capaz de classificar corretamente artigos científicos por campo com um alto grau de precisão com base em seus resumos. Porem, suas aplicações concretas, quanto à utilidade do procedimento para os administradores é discutida e oferece uma visão sobre a distribuição, concentração e sobreposição de recursos intelectuais. Além das aplicações práticas para o conteúdo científico indexação, diferenciação a semântica latente que poderão ser utilizadas para promover um programa de pesquisa científica examinando padrões de citação na base das características espaciais do índice.

E pode ser utilizada para obter as variáveis capazes de testar se a demografia espacial dos artigos pode ser responsável por variações em suas frequências de citação e ser aplicado a qualquer sistema representável em termos de dados textuais, avaliar as mudanças e padrões dentro do sistema ao longo do tempo, e oferecer informações valiosas sobre os processos de seleção e transformação que os caracterizam, sendo mais uma análise crítica sob as formas de representação dos índices de citação, fixando seu olhar restrito neste universo.

Já o quarto artigo, Contributions of Turkish academicians supervising PhD dissertations and their universities to economics: an evaluation of the 1990-2011 period, disponível na base de dados PROQUEST/LISA,_analisa as teses de doutorado realizadas nas universidades turcas no período de 1990-2011 e, as publicações indexadas pelos professores que supervisionaram tais teses. Incluídas publicações do Google Scholar, Web of Science e Scopus e as citações pertencentes às publicações. 
Totalizaram 617 professores que supervisionaram as 1.906 teses de doutorado no campo da economia levantadas. Referenciaram a Universidade de Istambul, Universidade de Marmara, Dokuz Eylül University, Ihsan Dogramaci Bilkent University, East Technical University Oriente e Universidade de Bogazici. Onde, em seus resultados indicaram que existem relações positivas e significativas com doutorados no exterior e o número de publicações na Web of Science.

Apontaram que as contribuições destas dissertações para a ciência internacional são discutidas. Na tentativa de determinar o nível de contribuição dos supervisores de dissertação para a economia e os dados utilizados para o estudo é limitado devido aos dados recolhidos a partir das dissertações concluídas nas universidades turcas. A metodologia apresentada no estudo incluíram técnicas estatísticas, diferentes tabelas e gráficos das universidades e professores com suas publicações.

Apresentaram dissertações realizadas nos institutos de ciências sociais das universidades oferecidos aos programas de doutorado em economia, bem como, as performances dos acadêmicos que realizaram estas teses. Foi possível identificar que os acadêmicos turcos estão atrás no mundo acadêmico em termos de número de publicações por professor e o número de citações destas publicações por diversos fatores.

E, que publicar nas revistas com reconhecimento internacional exige um esforço sem retorno financeiro e indica que a obtenção de um grau de doutorado no exterior tem um efeito positivo no que tange publicar na Web of Science Journal comparado a obtenção do grau de Doutor em uma universidade turca. Este estudo foi sob um olhar exclusivo do desempenho da universidade ora citada e abre uma reflexão para aplicações em outras instituições sob a mesma perspectiva, para que se possa de fato mensurar a colocação no ranking do mundo acadêmico.

A quinta análise se refere ao artigo Multiple h-index: a new scientometric indicator também disponível na base de dados PROQUEST/LISA, que avaliou alguns índices científicos, utilizando dados virtuais e propôs um novo índice, denominado múltipla $h$-index (mh-index).

As análises estatísticas, os coeficientes de correlação e diagramas comparativos relacionados mostraram que o mh-índice é mais preciso do que os outros nove variantes em diferenciar o impacto científico de pesquisadores com o mesmo índice h. Partiram da ideia que a sociedade científica precisa de um índice que avalie com precisão a produção científica do pesquisador individual.

E descreveram o relatório citação (o número de artigos e citação, autocitação, significa índice h) selecionados aleatoriamente com H-índices, registados numa lista de controle para o estudo. A categorização mostrou que os índices poderiam ser categorizados quanto ao seu núcleo produtivo. $\mathrm{O}$ artigo foi fundamentado para que o mundo científico use e avalie a saída 
da produção científica, podendo ser uma ferramenta de avaliação para pesquisadores para a sua necessidade científica mas, apresenta um alto nível de complexidade em sua aplicação e categorização, tornando difícil a replicação do estudo.

O sexto artigo, The Subject Sameness Index: A new scientific indicator da base de dados PROQUEST/LISA, aborda os indicadores cienciométricos para avaliação quantitativa e qualitativa das produções científicas e, demonstra que os indicadores têm forças e limitações, propondo um novo indicador cienciométrico $(S S I)$.

O estudo foi realizado num período de seis anos (2005-2010) analisando todos os artigos publicados por pesquisadores da Universidade de Babol de Ciências Médicas, que foram os primeiros autores com pelo menos dois papéis listados na Web of Science (WoS). Suas conclusões apresentam que a quantidade de publicações, citações e suas combinações nem sempre são os indicadores adequados de qualidade do trabalho científico.

O indicador SSI, pode acrescentar informações importantes de interesse para os administradores de pesquisa e editores científicos, qual também auxilia a formulação de políticas científicas, facilitando a comunicação científica, e desenhando um mapa científico global. Os autores, apontam que atualmente os indicadores disponíveis não são adequados para medir alguns aspectos importantes da produção científica e, o foco do pesquisador sobre uma determinada categoria assunto.

Salientaram o aparecimento da mesmice de assuntos contidas nos artigos, artigos com a repetição dos sinônimos ou palavras-chave sujeitos a todas as palavras-chave utilizadas por um pesquisador. Apontaram a importância dos pesquisadores estarem alinhados à temática e nomenclatura das publicações vistas pela visualização da dispersão dos esforços dos pesquisadores. E que a correção do índice depende de quais bancos de dados são usados para recuperar documentos. Um artigo de fácil leitura, qual desperta os cuidados e a atenção qual os pesquisadores necessitam estar aliados se apresentando como um método a avaliação complementar no que se traduz a qualidade das produções científicas.

Finalizando as análises com o artigo Qualis Periódicos: indicador da política científica do Brasil?, disponível na base de dados $E B S C O$, que apresenta a intenção de compreender e analisar a forma como o Qualis e seus critérios participam da rotina editorial dos periódicos científicos brasileiros.

A apresentação do surgimento do Qualis, seu funcionamento e modificações. O estudo aponta um levantamento bibliográfico sobre o tema, entrevistas e estudos etnográficos referente aos editores dos periódicos científicos da área de Educação.

Analisaram o Qualis como um indicador científico para a construção de um conceito de qualidade das publicações, pontuaram questões da qualidade dos artigos, critérios de

\begin{tabular}{|c|c|c|c|c|c|}
\hline (C) RDBCI: Rev. Digit. Bibliotecon. Cienc. Inf. & Campinas, SP & v.16 & n.2 & p. 414-432 & maio/ago. 2018 \\
\hline
\end{tabular}


arbitragem dos textos, órgãos publicadores, origem dos trabalhos, periódicos e indexação, a importância da Capes.

E, demonstraram que o Qualis é utilizado como um indicador no auxílio de financiamentos, na orientação de pesquisadores, na submissão de trabalhos e na pesquisa, além de estimular os editores a elevar o padrão de qualidade dos seus periódicos onde os elementos de padronização, internacionalização e regularidade se apresentam como fatores que orientam a definição de qualidade na produção científica nacional, medida por indicadores como o Qualis, onde este, necessita de melhorias para manter o nível de qualidade.

Porém salientam que o Qualis promove o reconhecimento e mérito científico dos periódicos e interfere na política científica e a melhoria qualitativa dos pesquisadores e das instituições de ensino.

\subsection{Sugestões para novas pesquisas}

Após os resultados encontrados na operacionalização das etapas, foi possível identificar pontos de estudos no que se refere à Gestão da Informação: indicadores científicos, o que pode promover outras discussões vistas ao desenvolvimento de novos conhecimentos e abordagens. São estes:

A. Necessidade da definição de métricas de forma clara, pois foi possível identificar que a detecção das informações e seus indicadores é um procedimento necessário na etapa de evolução da gestão da informação institucional, provocando o controle das ocorrências acadêmicas e científicas;

B. Sugerem-se quanto à avaliação dos indicadores, que nos próximos estudos estes estejam relacionadas de forma clara e objetiva para uma maior compreensão das medidas;

C. Quanto à gestão das informações, foi observada a parte qualitativa e quantitativa das informações, com olhar estritamente de produtividade científica e não a gestão da instituição;

D. Alguns modelos são indicados para a análise em relação a um problema, ao invés de capacitar o uso dos mecanismos especificamente;

E. Referentes aos indicadores sugere-se que as análises estejam também relacionadas às características de cada instituição e a sua percepção;

F. Estudos das métricas na visão dos usuários;

G. Em relação aos modelos utilizados, indicar a melhoria dos serviços, citar quais os mecanismos e o posicionamento dos participantes;

H. Sugere-se que na próxima análise as avaliações estejam relacionadas aos impactos científicos; e formas de apoio na gestão;

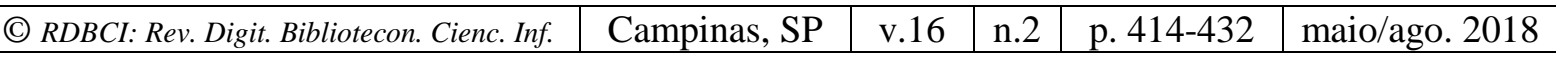


I. As análises poderão apresentar uma melhor descrição da qualidade e seus indicadores.

Pode se concluir, com base nas análises realizadas, que os estudos pertinentes à aplicação ou observação da Gestão da Informação: indicadores científicos podem ser ainda mais explorados, sendo na avaliação, reavaliação ou complementariedade das publicações existentes ou quanto às lacunas acima elencadas.

\section{CONCLUSÕES}

As análises possibilitaram identificar que não possuem indicadores explicitados para mensurar as medidas desempenho das instituições de ensino, suas metas e dimensões para posterior avaliação. São diversas e variadas formas de análise de produção científica, mas; não se obtiveram neste artigo trabalhos estritamente ligados a indicadores voltados as atividades e formas de trabalho das instituições de ensino superior (recursos x produção).

A revisão dos modelos de avaliação nos induz a pensar que existe uma grande lacuna para a pesquisa e formulação do estudo sobre o que seria os indicadores adequados para visualização a gestão da informação científica. Sendo assim, este artigo pode ser considerado uma premissa para a ocorrência de novos estudos.

Os estudos foram apresentados com análise em diversos países, realizou a análise de como têm sido tratadas as práticas da Gestão da Informação e os indicadores científicos no intuito de prover conhecimento e novos desafios e pesquisas para a área.

Das limitações, considerar periódicos disponibilizados no idioma inglês e espanhol, limitado a cinco bases de dados presentes no Portal da CAPES e, da intervenção do autor nas análises.

Foi possível observar dificuldades em relação à determinação dos indicadores científicos, na coleta de dados e registro das informações quais ficaram subentendidas em alguns artigos, falta de uma estrutura de um sistema de indicadores demonstrado de forma explícita.

Desta maneira, a relevância e importância de indicadores e medidas para a Gestão da Informação Científica nas Instituições de Ensino ainda se apresenta sem a notoriedade diante de um mercado em contínuo crescimento e visibilidade. Logo, para o auxílio e o atingimento dos objetivos institucionais, visualização da produção e aproveitamento dos usuários, controle dos processos, melhorias entre outros quesitos que se enquadram na gestão das instituições de ensino superior sugere-se novas abordagens e descrições sobre a Gestão da Informação: Indicadores Científicos. 


\section{REFERÊNCIAS}

BEAL, Adriana. Gestão estratégica da informação: como transformar a informação e a tecnologia em fatores de crescimento e de alto desempenho nas organizações. São Paulo: Atlas, 2004.

CONLEY, Shannon Nicole et al. Acquisition of T-shaped expertise: an exploratory study. Social Epistemology, v. 31, n. 2, p. 165-183, 2017.

DAHLBERG, Ingetraut. Knowledge organization: a new science?. Knowledge organization, v. 33, n. 1, p. 11-19, 2006. Disponível em: <http://www.db.dk/bh/lifeboat_ko/CONCEPTS/knowledge_organization_Dahlberg.htm>. Acesso em: nove. 2017.

DE SORDI, José Osvaldo. Administração da informação: fundamentos e práticas para uma nova gestão do conhecimento. Saraiva, 2008.

GAMEIRO, Maria Cristina. Sistemas e metodologia de arquivos nas empresas. In: Sistemas e metodologia de arquivos nas empresas. Cenadem, 1986.

MELNYK, Steven A. et al. Is performance measurement and management fit for the future?. Management Accounting Research, v. 25, n. 2, p. 173-186, 2014.

MICHELI, Pietro; MANZONI, Jean-Francois. Strategic performance measurement: Benefits, limitations and paradoxes. Long Range Planning, v. 43, n. 4, p. 465-476, 2010.

NEELY, Andy; GREGORY, Mike; PLATTS, Ken. Performance measurement system design: a literature review and research agenda. International journal of operations \& production management, v. 15, n. 4, p. 80-116, 1995.

OTLEY, David. Extending the boundaries of management accounting research: developing systems for performance management. The British Accounting Review, v. 33, n. 3, p. 243$261,2001$.

PINTO, Adilson Luiz et al. Indicadores científicos na literatura em bibliometria e cientometria através das redes sociais. Brazilian Journal of Information Science, v. 1, n. 1, p. 58-76, 2007. Disponível em:<https://dialnet.unirioja.es/descarga/articulo/4366117/1.pdf>. Acesso em: 02/10/2017.

PORTAL DA EDUCAÇÃO. A importância da Gestão da Informação. 2012. Disponível em: <https://www.portaleducacao.com.br/conteudo/artigos/esporte/a-importancia-da-gestao-dainformacao/15645>. Acesso em: 02/10/2017.

RODRIGUES, Martius V.; FERRANTE, Augustin J. A tecnologia de informação. Rio de Janeiro: Infobook, 1995.

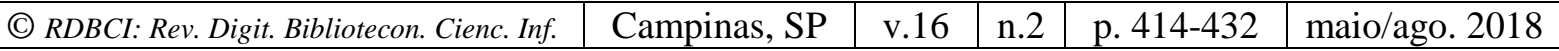


SANCHES, Osvaldo Maldonado. Dicionário de orçamento, planejamento e áreas afins. Prisma, 1997.

SINK, Scott; TUTTLE, Thomas C. Planejamento e medição para a performance. Qualitymark, 1993.

SPINAK, Ernesto. Diccionario enciclopédico de Bibliometría. Cienciometría e Informetría, 1996.
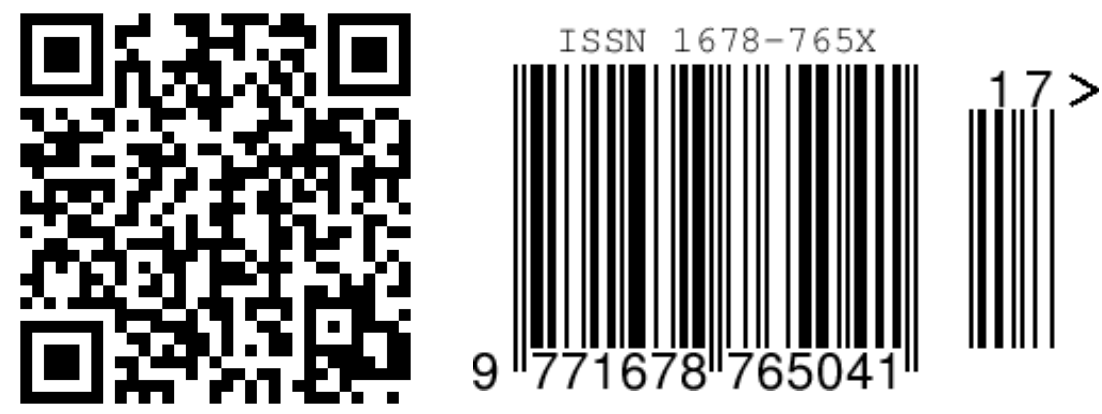